Pacific Journal of Mathematics

MAPPINGS OF BOUNDED CHARACTERISTIC INTO 


\title{
MAPPINGS OF BOUNDED CHARACTERISTIC INTO ARBITRARY RIEMANN SURFACES
}

\author{
D. J. H. FULLER
}

Introduction. Throughout this paper we consider analytic mappings $f(z)$ of an arbitrary open Riemann surface $R$ into an arbitrary Riemann surface $S$. Heins [3] introduced the class of Lindelöfian maps when $R$ is hyperbolic, and defined them in terms of Green's functions; further contributions have been made by Rao [4], [5]. In the case of planar regions these maps are the classical functions of bounded characteristic.

Sario [6], [7], has utilized principal functions [1] on the range surface to obtain generalizations of the main theorems for mappings of $R$ into $S$. In this paper a different first main theorem is obtained in which the proximity function is a generalization of Nevanlinna's proximity function by means of the substitution of a principal function for the logarithmic function. It is shown that the resulting class of functions of bounded characteristic are the Lindelöfian maps, and that an extremal decomposition characterization of these functions can be obtained as in the classical case.

1. An auxiliary family of functions. Analytic mappings from an arbitrary open surface $R$ into an arbitrary surface $S$ can be considered in terms of families $\mathscr{T}$ of $L H$ functions, i.e., harmonic functions, with isolated logarithmic singularities having integral coefficients. For the purposes of this paper we slightly generalize the term, parametric disk: $\quad \Delta=(Q, \mu)$ is a parametric disk if $Q$ is a classical parametric disk, and there is defined on it a metric $\mu$ that is a real scalar multiple of the induced metric.

We let $\zeta$ be the local variable on $S$, and fix $\sigma \in S$ and a parametric disk at $\sigma$. If $S$ is closed we define $t(\zeta, \sigma, \alpha)$ for $\alpha \in S \backslash \sigma$ (set difference) as the $L H$ function on $S$ which has singularities $\log |\zeta-\alpha|$ and $-\log |\zeta-\sigma|$ and is normalised by

$$
\lim _{\xi \rightarrow \sigma}(t(\zeta, \sigma, \alpha)+\log |\zeta-\sigma|)=0
$$

in terms of the fixed parametric disk. At $\alpha$ a parametric disk is fixed such that

$$
\lim _{\xi \rightarrow \infty}(t(\zeta, \sigma, \alpha)-\log |\zeta-\alpha|)=0
$$

Received September 19, 1963. This paper represents part of a thesis submitted to the faculty of the University of California, Los Angeles, in partial fulfillment of the requirements for the $\mathrm{Ph}$. D. degree. The author is indebted to Professor L. Sario for his guidance and to Dr. K. V. R. Rao for his advice and help. 
in terms of it. We define successively

$$
\begin{array}{cc}
t(\zeta, \alpha, \sigma)=-t(\zeta, \sigma, \alpha), & \alpha \in S \backslash \sigma, \\
t(\zeta, \alpha, \delta)=t(\zeta, \alpha, \sigma)+t(\zeta, \sigma, \delta), & \alpha, \delta \in S \backslash \sigma .
\end{array}
$$

These functions form the family $\mathscr{T}$.

If $S$ is open, more than one family can usually be formed. We consider an exhaustion of $S$ by regular regions $\Omega$ that contain $\sigma$ and $\alpha$, and define on $\Omega$ the function $t_{\Omega}(\zeta, \sigma, \alpha)$ which satisfies the above conditions for $t(\zeta, \sigma, \alpha)$ as well as one of the following:

(a) the normal derivative of $t_{\Omega}(\zeta, \sigma, \alpha)$ vanishes on the boundary $\partial \Omega$ of $\Omega$,

(b) a consistent partition of the boundaries of the regions $\Omega$ is given, and $t_{\Omega}(\zeta, \sigma, \alpha)$ has constant value and vanishing flux over each part of $\partial \Omega([1] \mathrm{pp} .87-90)$.

By the theory of normal operators ([1] pp. $152 \mathrm{ff}$.) $t(\zeta, \sigma, \alpha)$ is defined as the directed limit of $t_{\Omega}(\zeta, \sigma, \alpha)$ as $S$ is exhausted by the regions $\Omega . t(\zeta, \alpha, \sigma)$ and $t(\zeta, \alpha, \delta)$ are then defined as in the case of closed surfaces $S$. Each condition in (a) and (b) determines a family $\mathscr{T}$. It will be represented by $\mathscr{T}_{0}$ if (a) is satisfied and by $\mathscr{T}_{1}(P)$ if $(b)$ is satisfied for a partition $P$; if $P$ is the identity partition $I$, we write $\mathscr{T}_{1}(I)$.

Since each function $t$ is a principal function ([1] p. 169), a family $\mathscr{T}$ will be called a principal family. We note that a change in the fixed parametric disk at $\sigma$ changes every function $t(\zeta, \sigma, \alpha)$ by the same constant but leaves $t(\zeta, \alpha, \delta)$ unaltered. Further, in view of our definition of parametric disk, for any given $\mathscr{T}$ and constant $k$ there exists a family $\mathscr{T}^{\prime}$ such that for all $m$,

$$
\{\zeta \mid t(\zeta, \sigma, \alpha)=m\}=\left\{\zeta \mid t^{\prime}(\zeta, \sigma, \alpha)=m+k\right\}, t \in \mathscr{T}, t^{\prime} \in \mathscr{T}^{\prime} \text {. }
$$

We consider functions belonging to any principal family. If $\alpha, \delta \in S \backslash \sigma$, these functions have the following four obvious properties.

$$
\begin{aligned}
t(\zeta, \alpha, \alpha) & =0, \\
t(\zeta, \alpha, \delta)+t(\zeta, \delta, \alpha) & =0, \\
\lim _{\zeta \rightarrow \delta}(t(\zeta, \delta, \alpha)+\log |\zeta-\delta| & =t(\delta, \sigma, \alpha), \\
t(\sigma, \alpha, \delta) & =0 .
\end{aligned}
$$

Lemma 1.1. $t(\alpha, \gamma, \delta)+t(\gamma, \delta, \alpha)+t(\delta, \alpha, \gamma)=0$ when $\alpha, \gamma, \delta$ are distinct points in $S$.

Proof. If $S$ is open we let $\Omega \subseteq S$ be a regular region containing $\alpha, \gamma$, and $\delta$, and consider functions $t_{\Omega}$ defined on $\Omega$. We remove small closed disks in $\Omega$ that contain $\alpha, \gamma, \delta$ and apply Green's formula to 
$t_{\Omega}(\zeta, \alpha, \gamma)$ and $t_{s}(\zeta, \delta, \gamma)$ over the remaining region. On letting the disks shrink to points we obtain

$$
-t_{\Omega}(\alpha, \delta, \gamma)+t_{\Omega}(\delta, \alpha, \gamma)+R(\gamma)=0
$$

where

$$
R(\gamma)=\lim _{\zeta \rightarrow \gamma}\left(t_{\Omega}(\zeta, \delta, \gamma)-t_{\Omega}(\zeta, \alpha, \gamma)=t_{\Omega}(\gamma, \delta, \alpha)\right)
$$

and the lemma follows by letting $\Omega \rightarrow S$.

If $S$ is closed the same method is applied to $S$ instead of to $\Omega$.

Corollary 1.2. $t(\alpha, \delta, \sigma)=t(\delta, \alpha, \sigma), \alpha, \delta \in S \backslash \sigma$.

Proof. This is obvious when $\alpha$ and $\delta$ are identical; if they are distinct it follows from replacing $\gamma$ by $\sigma$ in the lemma and applying (1).

CoROLLARY 1.3. If $\alpha$ is distinct from $\sigma$ and $\gamma$, then $t(\alpha, \zeta, \gamma)$ is of class $L H$ on $S \backslash \sigma$ with singularities at $\alpha$ and $\sigma$.

Proof. If $\gamma=\sigma$ this is implied by Corollary 1.2. Otherwise

$$
t(\alpha, \zeta, \gamma)+t(\zeta, \sigma, \alpha)=t(\alpha, \sigma, \gamma),
$$

which is constant.

LEMMA 1.4. $\psi: S \backslash \sigma \times S \backslash \sigma \rightarrow[-\infty, \infty] \mid \psi(\gamma, \alpha)=t(\delta, \gamma, \alpha)$ is continuous for every fixed $\delta$.

Proof. If $\delta=\sigma$ then $\psi$ is identically zero; if not,

$$
\psi(\gamma, \alpha)=t(\delta, \gamma, \sigma)+t(\delta, \sigma, \alpha)
$$

and each term is continuous by Corollary 1.2.

Sario [8] proves that if $E \cong S \backslash \sigma$ is compact and $Q$ is an open set containing $E$ and $\sigma$, then $t(\gamma, \alpha, \sigma) \in \mathscr{T}_{0}$ is uniformly bounded for $\alpha \in E, \gamma \in S \backslash Q$. The same proof holds for $t(\gamma, \alpha, \sigma) \in \mathscr{T}_{1}(P)$. From the harmonicity of $t(\gamma, \alpha, \sigma)$ in $\gamma$ and in $\alpha$, and from its uniform boundedness, it follows by a lemma of Heins ([2] p. 445) that

Lemma 1.5. If $S^{\prime}=S \times S \backslash((\sigma, \sigma) \cup\{(\sigma, \zeta)\} \cup\{(\zeta, \sigma)\} \cup\{(\zeta, \zeta)\})$, then $\phi: S^{\prime} \rightarrow(-\infty, \infty) \mid \phi(\gamma, \alpha)=t(\gamma, \alpha, \sigma)$ is continuous.

Lemma 1.6. If $S^{\prime}=S \times S \backslash(\sigma, \sigma)$ then $\phi: S^{\prime} \rightarrow[-\infty, \infty] \mid \phi(\gamma, \alpha)=$ $t(\gamma, \alpha, \sigma)$ is continuous.

Proof. It suffices to consider the continuity at points $\left(\gamma_{0}, \alpha_{0}\right), \gamma_{0}=$ 
$\alpha_{0} \neq \sigma$, and $(\sigma, \alpha), \alpha_{0} \neq \sigma$. For the first we let $\Delta$ be a parametric disk at $\alpha_{0}$ such that $\sigma \notin \bar{\Delta}$, and $F$ be a closed connected neighborhood of $\alpha_{0}$ that does not intersect $\partial \Delta$. For every $(\gamma, \alpha) \in F \times F$ there exists $\eta \in \partial \Delta$ such that $t(\gamma, \alpha, \sigma)>t(\eta, \alpha, \sigma)$, which is bounded by Sario's lemma for all $\eta \in \partial \Delta, \alpha \in F$. No generality is lost by taking $t(\eta, \alpha, \sigma)>0$ for all $\eta \in \partial \Delta, \alpha \in F$.

Let $\psi$ be a homeomorphism of $\Delta$ onto a closed disk in the plane, and $g$ the Green's function on this disk. By its extremal property

$$
t(\zeta, \alpha, \sigma)-g(\psi(\zeta), \psi(\alpha)) \geqq 0
$$

for $\zeta, \alpha \in F$. Since for any $n$, there exists a neighborhood $E_{n}$ of the origin of the disk such that $g(z, a) \geqq n$ for $z, a \in E_{n}$, we have $t(\gamma, \alpha, \sigma)>n$. for $\gamma, \alpha \in \psi^{-1}\left(E_{n}\right) \cap F$, and $\phi$ is continuous at $\left(\gamma_{0}, \alpha_{0}\right)$.

For the second case we let $\partial \Delta$ be the boundary of a parametric disk at $\alpha_{0}$, and $F$ and $G$ be compact connected neighborhoods of $\sigma$ and $\alpha_{0}$ that do not intersect $\partial \Delta$. For $\zeta \in F, \alpha \in G$, there exists $\eta \in \partial \Delta$ such that

$$
t\left(\zeta, \alpha, \alpha_{0}\right)<t\left(\eta, \alpha, \alpha_{0}\right)=t(\eta, \alpha, \sigma)+t\left(\eta, \sigma, \alpha_{0}\right),
$$

which, by Sario's lemma, is bounded above, say by M. Hence

$$
t(\zeta, \alpha, \sigma)<M+t\left(\zeta, \alpha_{0}, \sigma\right),
$$

and the lemma follows, since for any $n, t\left(\zeta, \alpha_{0}, \sigma\right)<n$ in some neighborhood of $\sigma$.

We conclude this section by noting that the limits of $t(\zeta, \gamma, \sigma)$, $t(\zeta, \sigma, \gamma)$ and $t(\zeta, \gamma, \gamma)$ as $\gamma \rightarrow \sigma$ are $\infty,-\infty$ and 0 respectively, and that $t(\zeta, \sigma, \sigma)$ is not defined.

2. Jensen's formula. The main tool used in this paper is Jensen's: formula generalized for Riemann surfaces. We let $\Omega$ be a regularly imbedded relatively compact region on the surface $R$ and let $v(z)$ be an $L H$ function on $\bar{\Omega}$. The positive singularities of $v(z)$ in $\Omega$ will be designated by $a_{i}, i=1, \cdots, m$, and the negative singularities by $b_{j}, j=1, \cdots, n$; their multiplicities will be given by $\mu_{i}$ and $\nu_{j}$ respectively.

We obtain the formula from the following proposition:

LEMMA 2.1. If $r$ is not a singularity of $v(z)$, then

$$
v(r)=\frac{1}{2 \pi} \int_{\partial \Omega} v(z) d^{*} p(z, r)+\sum_{i} \mu_{i} g\left(a_{i}, r\right)-\sum_{j} \nu_{j} g\left(b_{j}, r\right)
$$

where $p(z, r)$ and $g(z, r)$ are the capacity and Green's functions defined on $\Omega$ with singularities at the point $r$, and $\partial \Omega$ is oriented counterclockwise about $r$. 
Proof. We first take the case when $v(z)$ has no singularities on $\bar{\Omega}$. Let $\Delta \subseteq \Omega$ be a small closed disk that contains $r$. On applying Green's formula to $p(z, r)$ and $v(z)$ over $\Omega \backslash \Delta$, and letting $\Delta$ shrink to $r$ we obtain

$$
v(r)=\frac{1}{2 \pi} \int_{\partial \Omega} v(z) d^{*} p(z, r)
$$

We next take the case when $v(z)$ has a singularity $\nu \log |z-a|$, $a \in \partial \Omega$, but has none in $\Omega$. Let $p(z, r)$ have the value $k$ on $\partial \Omega$; there exists an $\varepsilon>0$ such that the boundary components of $\Omega_{\varepsilon}=\{z \mid p(z, r)<k-\varepsilon\}$, have a natural one-to-one mapping on those of $\partial \Omega$.

Let the components of $\partial \Omega$ be $\left\{\gamma_{i}\right\}, i=1, \cdots, n$, with $a \in \gamma_{1}$, and the corresponding components of $\partial \Omega_{\varepsilon}$ be $\left\{\gamma_{i \varepsilon}\right\}$. For $i \neq 1$, we apply Green's formula to $v(z)$ and $p(z, r)$ over each component of $\Omega \backslash \Omega_{\varepsilon}$ and obtain

$$
\int_{\gamma_{i}-\gamma_{i \varepsilon}} v(z) d^{*} p(z, r)=\varepsilon \int_{\gamma_{i \varepsilon}} d^{*} v(z)
$$

For $i=1$, we let $\hat{\Omega}$ be the double of $\bar{\Omega} \backslash \Omega_{\varepsilon}$. If $q$ is the total flux of $p(z, r)$ along $\gamma_{1}$, the function

$$
h(z)=\exp \left[\frac{2 \pi}{q}\left(p(z, r)-k+i p^{*}(z, r)\right]\right.
$$

maps the first component of $\hat{\Omega}$ conformally onto an annulus, such that $\gamma_{1}$ is mapped onto the unit circle $B_{1}$ and $\gamma_{1 \varepsilon}$ onto $B_{1 \varepsilon}=\{w|| w \mid=$ $\exp [-(2 \pi / q) \varepsilon]\}$. We may assume that the point $a$ is mapped on $w=1$. Consequently $d \theta=2 \pi / q d^{*} p(z, r)$.

Since $\int_{0}^{2 \pi} \log \left|e^{i \theta}-1\right|=0$, it follows that

$$
\int_{\gamma_{1}-\gamma_{I \varepsilon}} v(z) d^{*} p(z, r)=\frac{q}{2 \pi} \int_{B_{1}-B_{1 \varepsilon}}\left(v \cdot h^{-1}\left(r e^{i \theta}\right)-\nu \log \left|r e^{i \theta}-1\right|\right) d \theta .
$$

By applying Green's formula to the last integrand and to $\log \left|r e^{i \theta}\right|$ over the annulus between the circles, we find

$$
\int_{\gamma_{1}-\gamma_{1 \varepsilon}} v(z) d^{*} p(z, r)=\varepsilon \int_{\gamma_{1 \varepsilon}} d^{*} v(z)
$$

Summing over all the components of $\partial \Omega$ we obtain

$$
\frac{1}{2 \pi} \int_{\partial \Omega} v(z) d^{*} p(z, r)=\frac{1}{2 \pi} \int_{\partial \Omega_{\varepsilon}} v(z) d^{*} p((z, r)=v(r) \text {. }
$$

For the general case we note that

$$
v(z)-\sum_{i} \mu_{i} g\left(\alpha_{i}, z\right)+\sum_{j} \nu_{j} g\left(b_{j}, z\right)
$$


is a harmonic function on $\Omega$. The application of (3) to this function ryields (2). We immediately obtain

CoRollary 2.2. (Generalized Jensen's formula). If $f$ is an analytic mapping of $R$ into a Riemann surface $S$ on which is defined a function $t(\zeta, \alpha, \delta)$ belonging to a principal family, and if $f(r), \alpha$ and $\delta$ are distinct, then,

$$
\begin{aligned}
t(f(r), \alpha, \delta)= & \frac{1}{2 \pi} \int_{\partial \Omega} t(f(z), \alpha, \delta) d^{*} p(z, r) \\
& \quad+\sum_{i} \mu_{i} g\left(a_{i}, r\right)-\sum_{j} \nu_{j} g\left(d_{j}, r\right) .
\end{aligned}
$$

where $\left\{a_{i}\right\}$ and $\left\{b_{j}\right\}$ are the inverse images in $\Omega$ of $\alpha$ and $\delta$ respectively, and $\mu_{i}, \nu_{j}$ are their multiplicities.

If $f(r)$ is a singularity of $t(\zeta, \alpha, \delta)$ the following proposition holds:

LEMMA 2.3. If $f(r)=\alpha$, and if the Laurent expansion of $f(z)$ in the neighborhood of $r$ is $f(z)=\sum_{N}^{\infty} c_{i} z_{i}$ with respect to the parametric disks at $r$ and $\alpha$ fixed by $p(z, r)$ and $t(\zeta, \sigma, \alpha)$ respectively, then

$$
\lim _{z \rightarrow r}(N p(z, r)+t(f(z), \sigma, \alpha))=\log \left|\frac{1}{c_{N}}\right| .
$$

If $f(z)=\delta$, then, with the above expansion,

$$
\lim _{z \rightarrow r}(-N p(z, r)+t(f(z), \sigma, \alpha))=\log \left|\frac{1}{c_{N}}\right| .
$$

Proof. We shall use the same symbol $z$ for an arbitrary point on the surface and for its image under the mapping associated with the parametric disk under consideration. $t(f(z))$ and $p(z)$ will represent $t(f(z), \sigma, \alpha)$ and $p(z, r)$, and $l_{i}$, etc., constant coefficients. We set

$$
q(z)=\exp \left[t(f(z))+i t^{*}(f(z))\right] ;
$$

this is single-valued in a neighborhood of $r$.

If $f(r)=\sigma$, the expansion in that neighborhood is

$$
q(z)=\frac{1}{c_{N}} z^{-N}+-\sum_{N+1}^{\infty} l_{i} z^{i}
$$

Similarly, there is a neighborhood of $r$ in which

$$
r(z)=\exp \left[p(z)+i p^{*}(z)\right]
$$

can be expanded as 


$$
r(z)=z+\sum_{1}^{\infty} m_{i} z^{i}
$$

Hence

$$
\frac{1}{c_{N}}=\lim _{z \rightarrow r} q(z)(r(z))^{N}
$$

which yields the first conclusion. The second is proved in the same way. This concludes the proof.

If we let $\lambda$ equal $N$ or $-N$ according as $f(r)$ is $\sigma$ or $\alpha$, then the function

$$
t(f(z))+\lambda p(z)-\sum_{i}^{\prime} \mu_{i} g\left(s_{i}, z\right)+\sum_{j}^{\prime} \nu_{j} g\left(a_{i}, z\right)
$$

is harmonic on $\Omega$, when the summations are over the inverse images in $\Omega \backslash r$. On applying Jensen's formula (4) and substituting from (5) or (6) we obtain the alternative expression

$$
\log \left|\frac{1}{c_{N}}\right|=\frac{1}{2 \pi} \int_{\partial \Omega} t(f(z)) d^{*} p(z)+k \lambda+\sum_{i}^{\prime} \mu_{i} g\left(s_{i}, r\right)-\sum_{j}^{\prime} \nu_{j} g\left(a_{i}, r\right) .
$$

We shall need the following property of subharmonic functions:

LEMMA 2.4. Let $u$ be an u.s.c. function on a region $W$.

(i) If $u$ is subharmonic on $W$, then for every regular $\Omega$ whose closure is in $W$, and every $z \in \Omega$,

$$
u(z) \leqq \frac{1}{2 \pi} \int_{\partial \Omega} u(w) d^{*} p_{\Omega}(w, z) .
$$

(ii) If for every $z \in W$, there is a regular $\Omega$ such that

$$
u(z) \leqq \frac{1}{2 \pi} \int_{\partial h} u(w) d^{*} p_{\Omega}(w, z)
$$

over every level line oh of $p_{\Omega}(w, z)$, then $u$ is subharmonic on $W$.

Proof. To prove (i) we take an arbitrary $\Omega$ and $z \in \Omega$, and let $\left\{v_{n}\right\}$ be a descending sequence of continuous functions on $\partial \Omega$ tending to $u$. For any $w \in \partial \Omega$, we have by (2)

$$
\varlimsup_{z \rightarrow w} u(z) \leqq \lim _{z \rightarrow w} \frac{1}{2 \pi} \int_{\partial \Omega} v_{n}(w) d^{*} p(w, z)
$$

for all $v_{n}$. By applying the monotone convergence theorem and the maximum principle we obtain the desired result.

For (ii) we let $z_{0}$ be an arbitrary point in the region, and choose a parametric disk about $z_{0}$. In terms of the associated unit disk the 
hypothesis yields

$$
u\left(z_{0}\right) \leqq \frac{1}{2 \pi} \int_{0}^{2 \pi} u\left(z_{0}+r e^{i \theta}\right) d \theta
$$

for $0<r \leqq 1$. The subharmonicity of $u$ follows from the theory of functions on the plane.

We immediately obtain

COROLlaRY 2.5. If $u(z)$ is subharmonic on $\bar{\Omega}_{2}$ and $z \in \Omega_{1} \subseteq \Omega_{2}$ r then

$$
\int_{\partial \Omega_{1}} u(w) d^{*} p_{1}(w, z) \leqq \int_{\partial \Omega_{2}} u(w) d^{*} p_{2}(w, z),
$$

where $p_{i}(w, z), i=1,2$, is the capacity function on $\Omega_{i}$.

3. Argument principle. Using the same notation as before we let $\Omega$ be a relatively compact regularly imbedded open set in the surface $R$, and $n(\Omega, \alpha), n(\Omega, \delta)$ the number of inverse images (with multiplicities) in $\Omega$ of points $\alpha$ and $\delta$ in $S$ that are not on the image of $\partial \Omega$. We have

$$
\frac{1}{2 \pi} \int_{\partial \Omega} d^{*} t(f(z), \alpha, \delta)=n(\Omega, \delta)-n(\Omega, \alpha)
$$

where $\partial \Omega$ is oriented counter-clockwise, and $t$ belongs to any principal family.

This statement follows from removing small disks at each of the inverse images of $\alpha$ and $\delta$, applying Green's formula to $t(f(z), \alpha, \delta)$ over the remainder of $\Omega$, and taking the limit as the disks shrink to points.

We choose $r \in \Omega$. If $p(z, r)$ is the capacity function on $\Omega$, and $k$ is its value on $\partial \Omega$, we let $\Omega_{h}=\{z \in \Omega \mid p(z, r)<h\}$ and $\partial h$ be the boundary of $\Omega_{h}$.

THEOREM 3.1. If $\alpha$ and $\delta$ are not in the image of $\partial h$, and if $\alpha, \delta$ and $f(r)$ are distinct, then

$$
\frac{1}{2 \pi} \frac{d}{d h} \int_{\partial h} t(f(z), \alpha, \delta) d^{*} p(z, r)=n(h, \delta)-n(h, \alpha),
$$

where $n(h, \delta)$ and $n(h, \alpha)$ are the number of inverse images (with multiplicities) of $\delta$ and $\alpha$ in $\Omega_{h}$, and $t$ belongs to any principal family.

Proof. We let $t(f(z))$ and $p(z)$ represent $t(f(z), \alpha, \delta)$ and $p(z, r)$, $\left\{a_{i}\right\}$ and $\left\{d_{j}\right\}$ be the finite number of inverse images of $\alpha$ and $\delta$ in $\Omega_{h}$. 
There is a finite $h^{\prime}$ such that $\Omega_{h^{\prime}}$ does not contain any of these inverse images. We remove small disks about the $\alpha_{i}^{\prime} \mathrm{s}$ and $d_{j}^{\prime} \mathrm{s}$ and apply Green's formula to $t(f(z))$ and $p(z)$ over the remainder of $\Omega_{h} \backslash \bar{\Omega}_{h^{\prime}}$. After evaluating and letting the disks shrink to points we obtain

$$
\begin{aligned}
& \int_{\partial h-\partial h^{\prime}} t(f(z)) d^{*} p(z)+2 \pi\left[\sum_{i} \mu_{i} p\left(a_{i}\right)-\sum_{j} \nu_{j} p\left(d_{j}\right)\right] \\
& =h \int_{\partial h} d^{*} t(f(z))-h^{\prime} \int_{\partial h^{\prime}} d^{*} t(f(z)),
\end{aligned}
$$

since $p(z)$ is the capacity function on both $\Omega_{h}$ and $\Omega_{h^{\prime}}$. In this relationship $\mu_{i}$ and $\nu_{j}$ are the multiplicities of the corresponding inverse images. The differentiation of this equation yields

$$
\begin{aligned}
\frac{d}{d h} \int_{\partial h} t(f(z)) d^{*} p(z) & =\frac{d}{d h}\left[h \int_{\partial h} d^{*} t(f(z))\right] \\
& =\lim _{\varepsilon \rightarrow 0} \frac{1}{\varepsilon}\left[\varepsilon \int_{\partial h} d^{*} t(f(z))-(h-\varepsilon) \int_{\partial(h-\varepsilon)-\partial h} d^{*} t(f(z))\right] .
\end{aligned}
$$

Since the last term vanishes for sufficiently small $\varepsilon$, we substitute from (9) and obtain the required relationship.

We note that (10) is an invariant property of principal families.

4. Logarithmic capacities. A logarithmic capacity of a compact set $E$ properly contained in an arbitrary surface $S$ can be defined in relation to any principal family $\mathscr{T}$ if $\sigma \in S \backslash E$. We let $\mu$ be a regular positive unit mass distribution on $E$. Since $t(\zeta, \eta, \sigma)$ is l.s.c. on $E$, we define the logarithmic potential of $\mu$ relative to $\mathscr{T}$ as

$$
p_{\mu}(\eta)=\int_{E} t(\zeta, \eta, \sigma) d \mu(\zeta)
$$

on $S \backslash \sigma$. The following proposition carries over from the plane:

Lemma 4.1. The logarithmic potential $p_{\mu}(\eta)$ is harmonic on $S \backslash(E \cup \sigma)$ and superharmonic on $S \backslash \sigma$. In the neighborhood of $\sigma, p_{\mu}(\eta)-\log |\eta-\sigma|$ is bounded.

Proof. We let

$$
t_{n}(\zeta, \eta, \sigma)=\min \{n, t(\zeta, \eta, \sigma)\},
$$

and

$$
p_{\mu_{n}}(\eta)=\int_{E} t_{n}(\zeta, \eta, \sigma) d \mu(\zeta)
$$

By Lemmas 1.5 and $1.6, t_{n}(\zeta, \eta, \sigma)$ is continuous in $(\zeta, \eta), \zeta \in E, \eta \in S \backslash \sigma$, 
and as $E$ is compact there is for any arbitrary point $\eta_{0} \in S \backslash \sigma$ and $\varepsilon>0$, a neighborhood $\Delta$ of $\eta_{0}$ such that

$$
\left|t_{n}(\zeta, \eta, \sigma)-t_{n}\left(\zeta, \eta_{0}, \sigma\right)\right|<\varepsilon, \zeta \in E, \eta \in \Delta,
$$

It follows that $p_{\mu_{n}}(\eta)$ is continuous and $p_{\mu}(\eta)$ l.s.c. on $S \mid \sigma$.

Let $\Gamma$ be a disk about $\eta_{0}$ such that $\partial \Gamma$ is a level line of $t\left(\zeta, \eta_{0}, \sigma\right)$. We orient $\partial \Gamma$ clockwise about $\eta_{0}$. Since $t(\zeta, \eta, \sigma)$ is bounded below for all $\zeta \in E, \eta \in \partial \Gamma$, and $-t\left(\zeta, \eta_{0}, \sigma\right)$ is the capacity function on $\Gamma$, we have by Corollary 1.2 and (2),

$$
\frac{1}{2 \pi} \int_{\partial \Gamma} d^{*} t\left(\eta, \eta_{0}, \sigma\right) \int_{E} t_{n}(\zeta, \eta, \sigma) d \mu(\zeta)=\int_{E} t_{n}^{\prime}\left(\zeta, \eta_{0}, \sigma\right) d \mu(\zeta)
$$

where for each $\zeta, t_{n}^{\prime}(\zeta, \eta, \sigma)$ is the harmonic function in $\eta$ on $\Gamma$ with boundary values $t_{n}(\zeta, \eta, \sigma)$. Further, by superharmonicity, $t_{n}^{\prime}\left(\zeta, \eta_{0}, \sigma\right) \leqq$ $t\left(\zeta, \eta_{0}, \sigma\right)$ for each $\zeta$ and for all $n$. We substitute in the above equation and apply the monotonic convergence theorem as $n \rightarrow \infty$. We obtain

$$
\frac{1}{2 \pi} \int_{\partial \Gamma} d^{*} t\left(\eta, \eta_{0}, \sigma\right) \int_{E} t(\zeta, \eta, \sigma) d \mu(\zeta) \leqq \int_{E} t\left(\zeta, \eta_{0}, \sigma\right) d \mu(\zeta)
$$

and $p_{\mu}(\eta)$ is superharmonic by (7).

If $\eta_{0} \notin E \cup \sigma, \Gamma$ can be chosen such that $\bar{\Gamma} \leqq S \backslash(E \cup \sigma)$. Since $t(\zeta, \eta, \sigma)$, is harmonic on $\bar{\Gamma}$, the same method establishes the harmonicity of $p_{\mu}(\eta)$ on $S \backslash(E \cup \sigma)$ by (2) and the maximum principle.

To establish the final part of the lemma we need only note that. by Lemmas 1.5 and 1.6 there is a neighborhood $\Delta$ of $\sigma$ such that

$$
t(\zeta, \eta, \sigma)=t(\eta, \zeta, \sigma)=\log |\eta-\sigma|+h(\eta, \zeta), \zeta \in E, \eta \in \triangle \mid \sigma,
$$

where $h(\eta, \zeta)$ is bounded.

We deduce the following proposition:

CoROllaRY 4.2. If $\mu$ is as above and $f: R \rightarrow S$ is analytic, then, for a regular $\Omega \subset R$,

$$
\int_{E} d \mu(\zeta) \int_{\partial \Omega} t(f(z), \zeta, \sigma) d^{*} p(z, r)=\int_{\partial \Omega} d^{*} p(z, r) \int_{E} t(f(z), \zeta, \sigma) d \mu(\zeta) .
$$

where $t$ belongs to any principal family $\mathscr{T}$ and $p$ is the capacity function on $\Omega$. The iterated integral is either finite or $+\infty$.

Proof. There exists a closed disk $D \subseteq S$ about $\sigma$ such that

(a) $D \cap E$ is void,

(b) $t(\alpha, \zeta, \sigma)<0, \alpha \in D, \zeta \in E$ by 1.6 ,

(c) the intersection of $D$ and the image of $\partial \Omega$ consists of a finite number (possibly zero) of Jordan arcs $\beta_{i}, i=1, \cdots, n$, each of which 
passes through $\sigma$.

We divide $\partial \Omega$ into the inverse images $\gamma_{i}, i=1, \cdots, n$, of $\beta_{i}$, and the remainder $\gamma$.

On $\gamma$ the function $t(f(z), \zeta, \sigma)$ is uniformly bounded below for $\zeta \in E^{\prime \prime}$ and we may apply Fubini's theorem to

$$
\int_{E} d \mu(\zeta) \int_{\gamma} t(f(z), \zeta, \sigma) d^{*} p(z, r) \text {. }
$$

The integral is either finite or $+\infty$.

For each $i$, we exhaust $\beta_{i} \backslash \sigma$ by a sequence of compact sets $F_{j}$. By (c) the restriction of $d^{*} p(z, r)$ to $f^{-1}\left(F_{j}\right) \cap \gamma_{i}$ induces a positive mass function on $F_{j} \subseteq D \backslash \sigma$. Its logarithmic potential

$$
p_{\mu i j}(\zeta)=\int_{f^{-1}\left(F_{j}\right) \cap \gamma_{i}} t(f(z), \zeta, \sigma) d^{*} p(z, r)
$$

is harmonic on $S \backslash D$ by 4.1. By (b) the functions $p_{\mu_{i j}}$ form a decreasing. sequence; by Harnack's principle its limit

$$
p_{\mu_{i}}(\zeta)=\int_{\gamma_{i}} t(f(z), \zeta, \sigma) d^{*} p(z, r)
$$

is either $-\infty$, or harmonic on $S \backslash D$.

We may assume that $p(z, r)$ is zero on $\partial \Omega$; then $\exp \left\{p(z, r)+i p^{*}(z, r)\right\}$ (choosing any branch of $p^{*}$ ) maps $\gamma_{i}$ onto an arc of the unit circle. For any $\zeta_{0} \in E$ we have

$$
p_{\mu_{i}}\left(\zeta_{0}\right)=\int_{\infty}^{\beta} \log \left|e^{i \theta}-1\right| d \theta+c
$$

where $c$ is some finite constant. Since this integral is bounded with respect to $\alpha$ and $\beta, p_{\mu_{i}}\left(\zeta_{0}\right)$ is finite, and $p_{\mu_{i}}(\zeta)$ is bounded on $E$.

Consequently, by (b), we may apply Fubini's theorem to

$$
\int_{E} d \mu(\zeta) \int_{\gamma_{i}} t(f(z), \zeta, \sigma) d^{*} p(z, r)
$$

for each $i$, and the integral is finite. Summing over $\gamma$ and $\gamma_{i}$, we obtain the required relation.

5. First main theorem. To develop a first main theorem for analytic mappings $f: R \rightarrow S$ where $R$ and $S$ are arbitrary Riemann surfaces, we fix a point $\sigma \in S$ and define a principal family $\mathscr{T}$; we then select points $\tau \in S$ and $r \in R$ such that $\sigma, \tau$ and $f(r)$ are distinct. A parametric disk is selected at $r$.

Let $\stackrel{\dot{t}}{t}=\max \{t, 0\}$. For a regular region $\Omega \equiv R$ such that $r \in \Omega$, the proximity function $m(\Omega, f)$, the counting function $N(\Omega, f)$ and the characteristic function $T(\Omega, f)$ of $f$ on $\Omega$ are defined as 


$$
\begin{aligned}
& m(\Omega, f)=\frac{1}{2 \pi} \int_{\partial \Omega}^{+} t(f(z), \sigma, \tau) d^{*} p(z, r), \\
& N(\Omega, f)=\sum_{i} g\left(s_{i}, r\right), \quad\left\{s_{i}\right\}=f^{-1}(\sigma) \cap \Omega, \\
& T(\Omega, f)=m(\Omega, f)+N(\Omega, f),
\end{aligned}
$$

where $p$ and $g$ are the capacity and Green's functions on $\Omega$, and $s_{i}$ is repeated in accordance with its multiplicity.

The proximity $m(\Omega, \alpha)$ and the counting functions $N(\Omega, \alpha)$ at the point $\alpha$ are defined as $m(\Omega, f)$ and $N(\Omega, f)$ when $\alpha=\sigma$; otherwise we define

$$
\begin{aligned}
& m(\Omega, \alpha)=\frac{1}{2 \pi} \int_{\partial \Omega}^{+}(f(z), \alpha, \sigma) d^{*} p(z, r), \\
& N(\Omega, \alpha)=\sum g\left(a_{i}, r\right), \quad\left\{a_{i}\right\}=f^{-1}(\alpha) \cap \Omega .
\end{aligned}
$$

where $a_{i}$ is repeated in accordance with its multiplicity.

The first main theorem reads:

THEOREM 5.1. For every $\alpha \in S \backslash f(r)$,

$$
m(\Omega, \alpha)+N(\Omega, \alpha)=T(\Omega, f)+0(1),
$$

where $O(1)$ is a bounded function with respect to $\Omega$.

Proof. When $\alpha=\sigma$ it is trivial; when $\alpha \neq \sigma$, Jensen's formula (4) is

$$
\begin{aligned}
t(f(r), \alpha, \sigma)= & \frac{1}{2 \pi} \int_{\partial \Omega}^{+} t(f(z), \alpha, \sigma) d^{*} p(z, r)+\sum_{i} g\left(a_{i}, r\right) \\
& -\frac{1}{2 \pi} \int_{\partial \Omega}^{+}(f(z), \sigma, \alpha) d^{*} p(z, r)-\sum_{i} g\left(s_{i}, r\right)
\end{aligned}
$$

which is

$$
\begin{aligned}
m(\Omega, \alpha)+N(\Omega, \alpha)= & \frac{1}{2 \pi} \int_{\partial \Omega}^{+}(f(z), \sigma, \alpha) d^{*} p(z, r) \\
& +N(\Omega, f)+O(1) .
\end{aligned}
$$

For $\zeta \in S$ we define

$$
q(\zeta)=\stackrel{+}{t}(\zeta, \sigma, \alpha)-\stackrel{+}{t}(\zeta, \sigma, \tau)
$$

There is a neighborhood $\Delta$ of $\sigma$ in which both $t(\zeta, \sigma, \alpha)$ and $t(\zeta, \sigma, \tau)$ are positive. Hence in 4 ,

$$
q(\zeta)=t(\zeta, \sigma, \alpha)-t(\zeta, \sigma, \tau)=t(\zeta, \tau, \alpha),
$$


which is bounded. Outside $\Delta, q(\zeta)$ is obviously bounded. It follows that

$$
\stackrel{+}{t}(f(z), \sigma, \alpha)=\stackrel{+}{t}(f(z), \sigma, \tau)+O(1) .
$$

We conclude the proof by substituting this in (12).

We note that if $\mathscr{T}^{\prime}$ and $\mathscr{T}^{\prime \prime}$ are principal families defined with respect to the same point $\sigma$, then the functions $t^{\prime}(\zeta, \sigma, \tau)$ and $t^{\prime \prime}(\zeta, \sigma, \tau)$ belonging to these families differ by a bounded harmonic function. Consequently the corresponding characteristic functtions $T_{1}$ and $T_{2}$ are related by $T_{1}(\Omega, f)=T_{2}(\Omega, f)+O(1)$ where $O(1)$ is bounded with respect to $\Omega$.

Before defining functions of bounded characteristic we shall develop an alternative representation of the characteristic function. For this purpose we prove the following lemma.

Lemia 5.2. $N(\Omega, \zeta)$ is continuous on $S \backslash f(r)$.

Proof. Let $\alpha$ be an arbitrary point in $S \backslash f(r)$, and let $a_{1}, \cdots, a_{q}$ with multiplicities $\nu_{1}, \cdots, \nu_{p}$ be the inverse images of $\alpha$ in $\Omega$.

We can construct open connected neighborhoods $D^{\prime}, D$ of $\alpha$ in $S \backslash f(r)$, and $E_{j}^{\prime}, E_{j}$ of $a_{j}$ in $\Omega \backslash r$ for every $j$, such that the following properties hold:

(a) Each neighborhood lies in a parametric disk about its associated point.

(b) Every inverse image of $\zeta \in D^{\prime} \backslash \alpha$ is simple and $\zeta$ has $\nu_{j}$ inverse images in $E_{j}^{\prime}$.

(c) Every $z \in E_{j}^{\prime} \backslash a_{j}$ is simple.

(d) $\bar{E}_{j} \subseteq E_{j}^{\prime}$.

(e) Every $\zeta \in D \backslash \alpha$ has $\nu_{j}$ roots in $E_{j}$, and $\bar{D} \subseteq D^{\prime}$.

$\left(E_{j}^{\prime} \backslash \alpha_{j}, f\right)$ is a smooth covering surface of $S$. If $\gamma(t)$ is an are in $D$ from an arbitrary $\delta \in D$ to $\alpha$, its path of determination $\gamma^{\prime}(t)$ from an inverse image of $\delta$ in $E_{j}$ cannot intersect $E_{j}^{\prime} \backslash E_{j}$ and must tend to $a_{j}$. Similarly if the inverse image is not in an $E_{j}^{\prime}, \gamma^{\prime}(t)$ must tend to $\partial \Omega$. Hence every component of the inverse image of $D$ that intersects $\Omega$ is either a neighborhood of some $a_{j}$ or intersects $\partial \Omega$.

Let $D_{0}=\left\{\xi|| \zeta-\alpha \mid<\rho_{0}\right\}$ be a disk in $D$ in terms of the local coordinates. Let $F_{j 0}$ be the component of the inverse image of $D_{0}$ that contains $a_{j}$, let $G_{j 0}, j=1, \cdots, n$ be the components that intersect the inverse images $b_{j}, j=1, \cdots, n$, of $\alpha$ on $\partial \Omega$, and let $H_{j 0}, j=1, \cdots, m$, be the other components that intersect $\Omega$. The number of components is finite since $\partial \Omega$ and $\partial D_{0}$ are analytic curves.

We define a real-valued function $h_{j}(z)$ on $H_{j 0}$ by $h_{j}(z)=|f(z)-\alpha|$. For each $H_{j 0}$ there exists $r_{j}>0$ such that $h_{j}(z)>r_{j}$ for $z \in H_{j 0} \cap \Omega$, 
and there exists a positive $r_{0}<r_{j}$ for all $j$. Let $D_{0}^{\prime}=\left\{\zeta|| \zeta-\alpha \mid<r_{0}\right\}$.

Let $M$ be a uniform bound of the number of inverse images in $\Omega$ of $\zeta \in S$. For $\varepsilon>0$ and every $j$, there exist neighborhoods $\Delta\left(b_{j}\right) \subseteq G_{j 0}$ of $b_{j}$ such that

$$
\left|g(z, r)-g\left(a_{j}, r\right)\right|<\frac{\varepsilon}{M}, \quad z \in \Delta\left(a_{j}\right),
$$

and

$$
|g(z, r)|<\frac{\varepsilon}{M}, \quad z \in \Delta\left(b_{j}\right)
$$

where $g(z, r)$ is the Green's function on $\Omega$ and vanishes outside $\Omega$. Then $|N(\Omega, \zeta)-N(\Omega, \alpha)|<\varepsilon$ in the intersection of $D_{0}^{\prime}$ and the images of $\Delta\left(a_{j}\right)$ and $\Delta\left(b_{j}\right)$ for all $j$. This completes the proof.

LEMMA 5.3. If $\mu$ is a regular positive unit measure on a compact set $E \subseteq S \backslash \sigma$, and if $p_{\mu}(\eta)=\int_{E} t(\eta, \zeta, \sigma) d \mu(\zeta)$ is the logarithmic potential with respect to any family $\mathscr{T}$, then

$$
\begin{aligned}
-p_{\mu}(f(r))= & -\frac{1}{2 \pi} \int_{\partial \Omega} p_{\mu}(f(z)) d^{*} p(z, r) \\
& +N(\Omega, f)-\int_{E} N(\Omega, \zeta) d \mu(\zeta) .
\end{aligned}
$$

Proof. By Lemmas 1.5 and 5.2 we may integrate Jensen's formula (4) over $E$ and obtain

$$
\begin{gathered}
\int_{E} t(f(r), \sigma, \zeta) d \mu(\zeta)=\frac{1}{2 \pi} \int_{E} d \mu(\zeta) \int_{\partial \Omega} t(f(z), \sigma, \zeta) d^{*} p(z, r) \\
+N(\Omega, f)-\int_{E} N(\Omega, \zeta) d \mu(\zeta)
\end{gathered}
$$

We apply (11) and obtain the required result, which is the natural generalization of Frostman's formula.

The characterization of $T(\Omega, f)$ that we need is a consequence of the next theorem.

For a fixed $\sigma, \tau \in S$ and $r \in R$ such that $\sigma, \tau$ and $f(r)$ are distinct, we shall write $t(\zeta)$ for $t(\zeta, \sigma, \tau), t_{m}(\zeta)$ for $\max \{m, t(\zeta)\}$ and $p(z)$ for $p(z, r)$.

THEOREM 5.4. If $E_{m}=\{\zeta \mid t(\zeta)=m\}$ where $m$ is finite and $t$ belongs to the principal family $\mathscr{T}_{1}(I)$ with respect to the identity partition, then 


$$
\begin{aligned}
t_{m}(f(r))= & \frac{1}{2 \pi} \int_{\partial \Omega} t_{m}(f(z)) d^{*} p(z) \\
& +N(\Omega, f)-\frac{1}{2 \pi} \int_{E_{m}} N(\Omega, \zeta) d^{*} t(\zeta) .
\end{aligned}
$$

Proof. We first prove this theorem for the case in which some extra hypotheses hold, and then remove the restrictions.

We assume that either $S$ is closed or that $S$ is a regular region containing the image of $\bar{\Omega}$ and that $m \neq \lim t(\zeta)$ as $\zeta \rightarrow \xi \in \partial S$. We choose a unit mass distribution on the compact set $E_{m}$ (oriented clockwise about $\sigma$ ) such that $d \mu=1 / 2 \pi d^{*} t(\zeta)$. Its logarithmic potential is

$$
p_{\mu}(\eta)=\frac{1}{2 \pi} \int_{E_{m}} t(\zeta, \eta, \sigma) d^{*} t(\zeta)
$$

$E_{m}$ divides $S$ into two components, one containing $\sigma$ and the other $\tau$; we shall call them the $\sigma_{m}$ and $\tau_{m}$-components. If $S$ is a regular region one of these components is a neighborhood of the ideal boundary; we suppose that it is the $\sigma_{m}$-component.

If $m<t(\eta)<\infty$, then the flux of $t(\zeta, \eta, \sigma)$ is zero over the boundary, $E_{m} \cup \partial S$, of the $\sigma_{m}$-component and is also zero over the boundary, $\partial S$, of $S$; since $E_{m} \cap \partial S$ is void, it follows that the flux over $E_{m}$ is zero. As $t(\zeta)$ is constant on $E_{m}$, it follows from (15) that

$$
-p_{\mu}(\eta)=-\frac{1}{2 \pi} \int_{E_{m}}\left[t(\zeta, \eta, \sigma) d^{*} t(\zeta)-t(\zeta) d^{*} t(\zeta, \eta, \sigma)\right] .
$$

The application of Green's formula to $t(\zeta, \eta, \sigma)$ and $t(\zeta)$ over the $\tau_{m}$-component proves that the right-hand side equals $-t(\tau, \eta, \sigma)=t_{m}(\eta)$.

If $-\infty<t(\eta)<m$, we write $t(\zeta, \eta, \sigma)=t(\zeta, \tau, \sigma)+t(\zeta, \eta, \tau)$ in (15). The flux of $t(\zeta, \tau, \sigma)$ is $2 \pi$ and the flux of $t(\zeta, \eta, \tau)$ is zero over the boundary, $E_{m}$, of the $\tau_{m}$-component. The first integral equals $-m$. We add a zero term and obtain

$$
-p_{\mu}(\eta)=m-\frac{1}{2 \pi} \int_{E_{m}}\left[t(\zeta, \eta, \tau) d^{*} t(\zeta)-t(\zeta) d^{*} t(\zeta, \eta, \tau)\right]
$$

from (15). We apply Green's formula to $t(\zeta, \eta, \tau)$ and $t(\zeta)$ over the $\sigma_{m}$-component, and it follows that $-p_{\mu}(\eta)=m-t(\sigma, \eta, \tau)=m=t_{m}(\eta)$ by (1).

We obtain the same results if we suppose that the $\tau_{m}$-component is a neighborhood of the ideal boundary.

Since the application of Lemma 4.1 to (15) shows that $p_{\mu}(\eta)$ is continuous at $\tau$, we conclude that $-p_{\mu}(\tau)=t_{m}(\tau)$.

If $\eta \in E_{m}$ we note that $t(\zeta, \eta, \sigma)$ is superharmonic in the neighborhood of $E_{m}$. We consider the level lines $E_{m-\varepsilon}$ and $E_{m+\varepsilon}, \varepsilon>0$. For sufficiently 
small $\varepsilon$, either $t(\zeta)$ is the capacity function on the $\tau_{m-\varepsilon^{-}}, \tau_{m^{-}}$and $\tau_{m+\varepsilon^{-}}$ components or $-t(\zeta)$ is the capacity function on the corresponding $\sigma$-components. In either case we apply (8) to (15) and obtain

$$
t_{m-\varepsilon}(\eta) \leqq-p_{\mu}(\eta) \leqq t_{m+\varepsilon}(\eta)
$$

which yields $-p_{\mu}(\eta)=t_{m}(\eta)$.

We substitute in (13) and obtain (14).

To remove the restrictions we shall denote the intersection of $E_{k}$ and the image of $\bar{\Omega}$ by $E_{k}^{\prime}$. Then $E_{k}^{\prime}$ is compact and

$$
\int_{E_{k}^{\prime}} N(\Omega, \zeta) d^{*} t(\zeta)=\int_{E_{k}} N(\Omega, \zeta) d^{*} t(\zeta) \text {. }
$$

If $S$ is a regular region and $m=\lim t(\zeta)$ as $\zeta \rightarrow \xi \in \partial S$, we take $\varepsilon_{0}>0$ sufficiently small that

$$
\{\zeta \mid \operatorname{grad} t(\zeta)=0 \text { and } m+\varepsilon \leqq t(\zeta) \leqq m\} \leqq E_{m} .
$$

For $\varepsilon_{0}>\varepsilon>0$ we map $E_{m+\varepsilon}^{\prime}$ into $E_{m}$ along the level lines of $t^{*}(\zeta)$. These are well defined as the different branches of $t^{*}(\zeta)$ differ by an additive constant. The mapping is one-to-one except that onto each of the finite number of zeros of grad $t(\zeta)$ on $E_{m}$ is mapped a finite number (one more than the order of the zero) of points on $E_{m+\varepsilon}^{\prime}$.

On the image of $\bar{\Omega}$ we set the measures $d \mu_{\varepsilon}=1 / 2 \pi d^{*} t(\zeta)$ on $E_{m+\varepsilon}^{\prime}, 0<\varepsilon<\varepsilon_{0}$. By Helly's theorem there exists a limiting measure that is obviously on $E_{m}^{\prime}$. By the continuity of the normal derivative of $t(\zeta)$ it is, under the above mapping, $d^{*} t(\zeta)$, a.e. Hence, if $N_{q}=$ $\min (N, q)$, we obtain

$$
\lim _{\varepsilon \rightarrow 0} \int_{E_{m+\varepsilon}^{\prime}} N_{k}(\Omega, \zeta) d^{*} t(\zeta) \leqq \int_{E_{m}^{\prime}} N(\Omega, \zeta) d^{*} t(\zeta)
$$

The opposite inequality is obtained by Fatou's lemma. Consequently,

$$
\lim _{\varepsilon \rightarrow 0} \int_{R_{m}+\varepsilon} N(\Omega, \zeta) d^{*} t(\zeta)=\int_{E_{m}} N(\Omega, \zeta) d^{*} t(\zeta) .
$$

We now establish (14) for $m$ by applying it to $m+\varepsilon$, which is permissible, and letting $\varepsilon \rightarrow 0$.

If $S$ is arbitrary we consider an exhaustion of $S$ by regular regions $W$ such that $W$ contains $\sigma, \tau$ and the image of $\bar{\Omega}$. We denote by $t_{W}(\zeta)=t_{W}(\zeta, \sigma, \tau)$ the function in the $\mathscr{T}_{1}(I)$ family defined with respect to $W$, and we set $E_{W m}=\left\{\zeta \in W \mid t_{W}(\zeta)=m\right\}$.

Let $W_{0}$ be a regular region containing the image of $\bar{\Omega}$. We first consider $m$ such that $E_{m} \cap \bar{W}_{0}$ contains no zeros of $\operatorname{grad} t(\zeta)$, and cover it with a finite number of parametric disks. We select $\varepsilon_{0}>0$, such that the set 


$$
F=\left\{\zeta \mid \varepsilon_{0} \leqq t(\zeta) \leqq m+\varepsilon_{0}\right\} \cap \bar{W}_{0}
$$

is contained in these disks and does not contain any zeros of grad $t(\zeta)$. On each disk we use $t(\zeta)$ and any branch of $t^{*}(\zeta)$ as local variables.

Since $E_{m}$ and $\partial W_{0}$ are analytic manifolds, their intersection consists of a finite number of components. Consequently there exists a compact $F^{\prime} \subseteq F$ such that the intersection of $E_{m}$ and the image of $\bar{\Omega}$ is contained in the interior of $F^{\prime}$, and that $\partial F^{\prime}$ intersects $E_{m}$ at a finite number of points, each of which has a neighborhood in which $\partial F^{\prime}$ lies on a level line of $t^{*}(\zeta)$. We set $E_{m}^{*}=E_{m} \cap F^{\prime}$

Since $t_{W}(\zeta)$ and its normal derivative tend uniformly on compact sets to $t(\zeta)$ and its normal derivative, there is for any $\varepsilon>0$, a $W_{\varepsilon}$ such that

$$
E_{W m} \leqq\{\zeta \mid m-\varepsilon<t(\zeta)<m+\varepsilon\} \cap F^{\prime},
$$

and that the maximum angle between $E_{W m}^{\prime}$ and $E_{m}$ is less than $\pi / 2$, for $W \supseteqq W_{\varepsilon}$. For sufficiently small $\varepsilon$ we can $\operatorname{map} E_{W m}^{*}$ univalently onto $E_{m}^{*}$ along the level lines of $t^{*}(\zeta)$.

We have set up the set we need for the proof. We apply (14) to the region $W$ and let $W \rightarrow S$. It is only necessary to examine the convergence of the last term. On $\bar{W}_{0}$ we choose a set of measures $d \mu_{W}=1 / 2 \pi d^{*} t_{W}(\zeta)$ on $E_{W m}^{*}$. For sufficiently large $W$,

$$
\int_{E_{W m}^{*}} N(\Omega, \zeta) d^{*} t_{W}(\zeta)=\int_{E_{W m}} N(\Omega, \zeta) d^{*} t_{W}(\zeta)
$$

We apply Helly's theorem as before and obtain the necessary convergence. Consequently, the theorem holds for open $S$ if there is no zero of grad $t(\zeta)$ on $E_{m}$.

If grad $t(\zeta)$ has a zero on $E_{m}$, we apply (14) to $E_{m+\varepsilon}$ and take the limit as $\varepsilon \rightarrow 0$. To obtain the convergence of the last term, we choose the set of measures $d \mu_{\varepsilon}=1 / 2 \pi d^{*} t(\zeta)$ on $E_{m+\varepsilon}^{*}$ and apply Helly's theorem. This completes the proof.

By taking $m=0$ in (14) we immediately obtain a generalization of Cartan's formula:

CoRollary 5.5. If the characteristic $T(\Omega, f)$ is defined in terms of a principal family $\mathscr{T}_{1}(I)$, then

$$
T(\Omega, f)=\stackrel{+}{t}(f(r))+\frac{1}{2 \pi} \int_{E_{0}} N(\Omega, \zeta) d^{*} t(\zeta) .
$$

As a side issue we shall strengthen Lemma 5.2.

LEMma 5.6. If $f(\partial \Omega)$ is the image of $\partial \Omega$, then $N(\Omega, \zeta)$ is $L P$ on $S \backslash f(\partial \Omega)$. 
Proof. Let $\alpha \in S \backslash(f(\partial \Omega) \cup f(r))$. We take $\sigma$ at $\alpha$, a parametric disk $\Delta$ at $\alpha$, and an arbitrary $\tau$. Let $t(\zeta)=t(\zeta, \sigma, \tau) \in \mathscr{T}_{1}(I)$. There exists $m_{0}$ such that $\left\{\zeta \mid t(\zeta) \leqq m_{0}\right\} \subseteq \triangle(f(\partial \Omega) \cup f(r))$.

For $m \geqq m_{0}$, (14) yields

$$
m=\frac{1}{2 \pi} \int_{\partial \Omega} m d^{*} p(z)+N(\Omega, \alpha)-\frac{1}{2 \pi} \int_{E_{m}} N(\Omega, \zeta) d^{*} t(\zeta),
$$

which is

$$
N(\Omega, \alpha)=\frac{1}{2 \pi} \int_{E_{m}} N(\Omega, \zeta) d^{*} t(\zeta)
$$

Since $-t(\zeta)$ is the capacity function on the neighborhood of $\alpha$ bounded by $m \geqq m_{0}$, the function $N(\Omega, \zeta)$ is harmonic on $S \backslash(f(\partial \Omega) \cup f(r)$.

Let the multiplicity of $r$ be $k$. By the construction used in Lemma 5.2 there is for any $n$, a neighborhood of $f(r)$ such that each $\zeta$ therein has $k$ inverse images in

$$
\{z \mid g(z, r)>n\},
$$

and a uniformly bounded number of other inverse images, for all of which $g(z, r)$ is uniformly bounded above. Hence $N(\Omega, \zeta)$ has a logarithmic singularity with coefficient $k$. This completes the proof.

6. Functions of bounded characteristic. The remark after Theorem 5.1 shows that if the characteristic function $T(\Omega, f)$ is bounded with respect to $\Omega$ when it is defined in terms of one principal family $\mathscr{T}$, then it is also bounded when defined in terms of another family. We shall show that this property is also independent of the points $\sigma, \tau, r$, provided that $\sigma, \tau$ and $f(r)$ are distinct.

For a fixed family $\mathscr{T}_{1}(I)$ and a fixed $\tau$, we define

$$
\begin{aligned}
x(\Omega, q) & =\frac{1}{2 \pi} \int_{\partial \Omega}^{+} t(f(z), \sigma, \tau) d^{*} p_{\Omega}(z, q), \\
y(\Omega, q) & =\sum_{i} g_{\Omega}\left(s_{i}, q\right), \quad\left\{s_{i}\right\}=f^{-1}(\sigma) \cap \Omega, \\
x^{\prime}(\Omega, q) & =\frac{1}{2 \pi} \int_{\partial \Omega}^{+} t(f(z), \tau, \sigma) d^{*} p_{\Omega}(z, q), \\
y^{\prime}(\Omega, q) & =\sum_{i} g_{\Omega}\left(t_{i}, q\right), \quad\left\{t_{i}\right\}=f^{-1}(\tau) \cap \Omega,
\end{aligned}
$$

and $u=x+y, u^{\prime}=x^{\prime}+y^{\prime}$, where $\Omega$ is a regular region in $R$, and $s_{i}$, $t_{i}$ are repeated in accordance with their multiplicities.

Lemma 6.1. If $\Omega$ exhausts $R$, then the limits of $y(\Omega, q)$ and 
$u(\Omega, q)$ are either LP (harmonic with positive logarithmic singularities) functions or $+\infty$; if the limit of $u(\Omega, q)$ is $L P$, then the limit of $x(\Omega, q)$ is harmonic.

Proof. The classical method is employed. We first prove that $\Omega_{1} \leqq \Omega_{2}$ implies $u\left(\Omega_{1}, q\right) \leqq u\left(\Omega_{2}, q\right)$. Let $z \in \bar{\Omega}_{1}$. We write $t(\zeta)$ for $t(\zeta, \sigma, \tau)$. If $t(f(z))>0$, then ${ }^{+}(f(z))=t(f(z))=u\left(\Omega_{2}, z\right)-u^{\prime}\left(\Omega_{2}, z\right)$ by Jensen's formula (14). Hence $\stackrel{+}{t}(f(z)) \leqq u\left(\Omega_{2}, z\right)$ for all $z \in \bar{\Omega}_{1}$. Consequently,

$$
\frac{1}{2 \pi} \int_{\partial \Omega_{1}}+(f(z)) d^{*} p_{1}(z, q) \leqq \frac{1}{2 \pi} \int_{\partial \Omega_{1}}\left[u\left(\Omega_{2}, z\right)-\sum_{i} g_{1}\left(s_{i}, z\right)\right] d^{*} p(z, q),
$$

which is, by transposition,

$$
u\left(\Omega_{1}, q\right) \leqq u\left(\Omega_{2}, q\right) .
$$

For any fixed $\Omega_{0}$ we exhaust $R$ by $\Omega \supseteqq \Omega_{0}$. By the application of Harnack's principle to $u(\Omega, q)-u\left(\Omega_{0}, q\right)$ over $\Omega_{0}$, we find that the limit of $u(\Omega, q)$ is $L P$ or $+\infty$ over $\Omega_{0}$ and hence over $R$.

By the maximum principle, $y\left(\Omega_{1}, q\right) \leqq y\left(\Omega_{2}, q\right)$ when $\Omega_{1} \subseteq \Omega_{2}$, and the same proof carries through.

If the limit of $u(\Omega, q)$ is $L P$, so is that of $y(\Omega, q)$ : further, both functions have the same singularities. By taking the limit of $x(\Omega, q)=$ $u(\Omega, q)-y(\Omega, q)$, we obtain the harmonicity of the limit of $x(\Omega, q)$. This completes the proof.

THEOREM 6.2. If $T(\Omega, f)$ is bounded with respect to $\Omega$, then it is bounded for any choice of $r, \tau, \sigma$ if $f(r), \sigma$ and $\tau$ are distinct.

Proof. A subscript indicates functions defined in terms of the new parameters.

(a) If $r$ is changed to $r_{1}$ such that $f\left(r_{1}\right) \neq \sigma$, then $T_{1}(\Omega, f)=$ $u\left(\Omega, r_{1}\right)$ is bounded since the limit of $u(\Omega, q)$ is $L P$.

(b) If $\tau$ is changed to $\tau_{1}$, we have

$$
\left|T_{1}(\Omega, f)-T(\Omega, f)\right| \leqq \frac{1}{2 \pi} \int_{\partial \Omega}\left|+t_{1}^{+}(f(z))-\stackrel{+}{t}(f(z))\right| d^{*} p(z, r) .
$$

The integrand is bounded since the function $q(\zeta)$ in the proof of Theorem 5.1 is bounded.

(c) If $\sigma$ is changed to $\sigma_{1}$, we may by (b) take $\sigma_{1}$ as $\tau$ in defining $T(\Omega, f)$. From the definitions of the terms

$$
T(\Omega, f)-\left(m_{1}(\Omega, \sigma)+N_{1}(\Omega, \sigma)\right)
$$

is a constant function. It follows from Theorem 5.1 that $T(\Omega, f)-$ $T_{1}(\Omega, f)=O(1)$. 
We have established the fact that the following class of functions is well-defined.

DEFinition. An analytic function $f: R \rightarrow S$, where $R$ is an arbitrary open surface and $S$ an arbitrary surface, is of bounded characteristic, $f \in M S$, if $T(\Omega, f)$ is bounded with respect to $\Omega \subseteq R$.

It follows from Lemma 6.1 that if $f \in M B$, then the limit of $y(\Omega, q)$ is a positive superharmonic function on $R$. Consequently $R$ is hyperbolic and we set

$$
N(R, \zeta, r)=\lim _{\Omega \rightarrow R} N(\Omega, \zeta)=\lim _{\Omega \rightarrow R} \sum_{i} g_{\Omega}\left(z_{i}, r\right), \quad\left\{z_{i}\right\}=f^{-1}(\zeta) \cap \Omega .
$$

Since ([3] p. 429) $N(R, \zeta, r)=\sum_{i} g\left(z_{i}, r\right),\left\{z_{i}\right\}=f^{-1}(\zeta)$, where $g$ is Green's function on $R$, it follows ([3] p. 418) that the class $M B$ is identical with the Lindelöfian maps. We are able to obtain a characterization in terms of $N(R, \zeta, q)$.

THEOREM 6.3. If $f: R \rightarrow S$ is analytic, the following statements are equivalent:

(a) $f \in M B$

(b) there exists $s \in R$ and open $U \subseteq S$ such that $N(R, \zeta, s)<\infty$ for $\zeta \in U$,

(c) $N(R, \zeta, s)<\infty, s \in R, \zeta \in S \backslash f(r)$.

Proof. To prove that (b) implies (a) we select $\alpha \in U \backslash f(s)$ and a parametric disk $\Delta$ at $\alpha$ such that $\bar{\Delta} \leqq U \backslash f(s)$.

Set $A_{n}=\left\{\zeta \in \bar{\Delta}_{n} \mid N(R, \zeta, s) \leqq n\right\}$ then by Lemma $5.2 N(R, \zeta, s)$ is lower semi-continuous and $A_{n}$ is closed. Also $\bar{\Delta}=\cup A_{n}$. By Baire's category theorem there exists $M$ such that $A_{M}$ has an interior point.

Let $Q \subseteq A_{M}$ be an open region, and $\tau \in Q \backslash(\sigma \cup f(s))$. We define a family $\mathscr{T}_{1}(I)$ at $\sigma ; t(\zeta, \sigma, \tau)$ has a level line $E$ in $Q$. There is a principal family $\mathscr{T}_{1}^{\prime}(I)$ such that $E=\left\{\zeta \mid t^{\prime}(\zeta)=0\right\}, t^{\prime} \in \mathscr{T}_{1}^{\prime}(I)$. Substitution in (16) yields

$$
T(\Omega, f) \leqq \stackrel{+}{t^{\prime}}(f(s))+\int_{E} M d^{*} t^{\prime}(\zeta)<\infty
$$

for all $\Omega$. Hence $f \in M B$.

(c) implies (b) trivially. To show that (a) implies (c) we note that by Lemma 6.1, $N(\Omega, \zeta)$ is bounded above for $s \in R, \zeta \in S \backslash f(s)$, whenever $f \in M B$. This completes the proof.

An extremal decomposition characterization of $M B$ functions is given by the following:

THEOREM 6.4. An analytic $f: R \rightarrow S$ is of class $M B$ if and only 
if $t(f(z))$ is the difference between two LP functions, where $t$ may be from any principal family $\mathscr{T}$.

Proof. From the proof of Lemma 6.1,

$$
t(f(z))=u(\Omega, z)-u^{\prime}(\Omega, z)
$$

for all $\Omega$. If $f \in M B$, then the limits of $u$ and $u^{\prime}$ are $L P$ functions. This proves the necessity.

For the sufficiency we assume $t(f(z))=v(z)-w(z), v, w \in L P$.

The singularities of $u(\Omega, z)$ are positive singularities of $t(f(z))$, and so among the singularities of $v(z)$. Hence $v(z)-u(\Omega, z)$ is superharmonic on $\Omega$ and attains its minimum on $\partial \Omega$.

Let $w \in \partial \Omega$. By $(2), x(\Omega, z)$ is the harmonic function on $\Omega$ with boundary values $t(f(w))$ and

$$
\lim _{z \rightarrow w} x(\Omega, z)=\stackrel{+}{t}(f(w))
$$

for any approach to $w$; also $y(\Omega, z) \rightarrow 0$ as $z \rightarrow w$, and $v(w) \geqq t(f(w))$. Consequently $v(z)-u(\Omega, z) \geqq 0$ on $\Omega$.

Since $v(z) \in L P$, there exists $r \in \Omega$ such that $v(r)<\infty$. Hence $u(\Omega, r)$ is bounded for all $\Omega$, and $f \in M B$. This concludes the proof.

The integrand of the proximity function used by Sario [8] is

$$
\begin{aligned}
s(\zeta, \alpha) & =t(\zeta, \alpha, \sigma)+\log \left(1+e^{2 t(\zeta)}\right)\left(1+e^{2 t(\alpha)}\right), \alpha \neq \sigma \\
& =\log \left(1+e^{2 t(\zeta)}\right), \quad \alpha=\sigma .
\end{aligned}
$$

where $t \in \mathscr{T}_{0}$ and $t(\zeta)=t(\zeta, \sigma, \tau)$. A comparison of the characteristic functions, evaluated at $\sigma$, shows that the functions of bounded characteristic with respect to Sario's characteristic function are the same as those treated above.

\section{BIBLIOGRAPHY}

1. L. Ahlfors L. Sario, Riemann surfaces, Princeton University Press, Princeton, 1960.

2. M. Heins, On the Lindelöf principle, Ann. of Math., (2) 61 (1955), 440-473.

3. —_ Lindelöfian maps, ibid. (2) 62 (1955), 418-446.

4. K. V. R. Rao, Lindelöfian maps and positive harmonic functions, Technical Report No. 28, ARO (D) Project 1517-M, April 1962, 48 pp.

5. - Positive harmonic functions, Lindelöfian meromorphic functions and Jensen's inequality, ibid. No. 31, 12 pp.

6. L. Sario, Meromorphic functions and conformal metrics on Riemann surfaces, Pacific J. Math., 12 (1962), 1079-1097.

7. - Value distribution under analytic mappings of arbitrary Riemann surfaces, Acta Math., 109 (1963), 1-10.

8. - Second main theorem without exceptional intervals on arbitrary Riemann surfaces, Mich. J. Math., 10 (1963), 207-219. 



\section{PACIFIC JOURNAL OF MATHEMATICS}

\section{EDITORS}

\author{
Robert Osserman \\ Stanford University \\ Stanford, California
M. G. Arsove
University of Washington
Seattle 5 , Washington

\author{
J. DugundJI \\ University of Southern California \\ Los Angeles 7, California
}

Lowell J. Paige

University of California

Los Angeles 24, California

\section{ASSOCIATE EDITORS}
E. F. BECKENBACH
B. H. NeumanN
F. WOLF
K. YOSIDA

\section{SUPPORTING INSTITUTIONS}

\author{
UNIVERSITY OF BRITISH COLUMBIA \\ CALIFORNIA INSTITUTE OF TECHNOLOGY \\ UNIVERSITY OF CALIFORNIA \\ MONTANA STATE UNIVERSITY \\ UNIVERSITY OF NEVADA \\ NEW MEXICO STATE UNIVERSITY \\ OREGON STATE UNIVERSITY \\ UNIVERSITY OF OREGON \\ OSAKA UNIVERSITY \\ UNIVERSITY OF SOUTHERN CALIFORNIA
}

\author{
STANFORD UNIVERSITY \\ UNIVERSITY OF TOKYO \\ UNIVERSITY OF UTAH \\ WASHINGTON STATE UNIVERSITY \\ UNIVERSITY OF WASHINGTON \\ * * * * \\ AMERICAN MATHEMATICAL SOCIETY \\ CALIFORNIA RESEARCH CORPORATION \\ SPACE TECHNOLOGY LABORATORIES \\ NAVAL ORDNANCE TEST STATION
}

Mathematical papers intended for publication in the Pacific Journal of Mathematics should by typewritten (double spaced), and on submission, must be accompanied by a separate author's résumé. Manuscripts may be sent to any one of the four editors. All other communications to the editors should be addressed to the managing editor, L. J. Paige at the University of California, Los Angeles 24, California.

50 reprints per author of each article are furnished free of charge; additional copies may be obtained at cost in multiples of 50 .

The Pacific Journal of Mathematics is published quarterly, in March, June, September, and December. Effective with Volume 13 the price per volume (4 numbers) is $\$ 18.00$; single issues, $\$ 5.00$. Special price for current issues to individual faculty members of supporting institutions and to individual members of the American Mathematical Society: $\$ 8.00$ per volume; single issues $\$ 2.50$. Back numbers are available.

Subscriptions, orders for back numbers, and changes of address should be sent to Pacific Journal of Mathematics, 103 Highland Boulevard, Berkeley 8, California.

Printed at Kokusai Bunken Insatsusha (International Academic Printing Co., Ltd.), No. 6, 2-chome, Fujimi-cho, Chiyoda-ku, Tokyo, Japan.

PUBLISHED BY PACIFIC JOURNAL OF MATHEMATICS, A NON-PROFIT CORPORATION

The Supporting Institutions listed above contribute to the cost of publication of this Journal, but they are not owners or publishers and have no responsibility for its content or policies. 


\section{Pacific Journal of Mathematics}

\section{Vol. 14, No. $3 \quad$ July, 1964}

Erik Balslev and Theodore William Gamelin, The essential spectrum of a class of ordinary differential operators . . . . . . . . . . . . . . . . . . . .

James Henry Bramble and Lawrence Edward Payne, Bounds for derivatives in

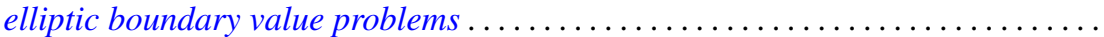

Hugh D. Brunk, Integral inequalities for functions with nondecreasing

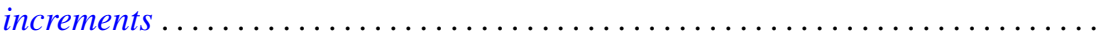

William Edward Christilles, A result concerning integral binary quadratic

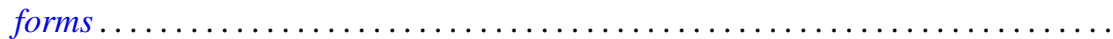

Peter Crawley and Bjarni Jónsson, Refinements for infinite direct decompositions of

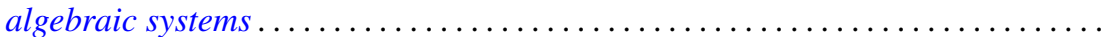

Don Deckard and Carl Mark Pearcy, On continuous matrix-valued functions on a Stonian space.

Raymond Frank Dickman, Leonard Rubin and P. M. Swingle, Another

characterization of the $n$-sphere and related results $\ldots \ldots \ldots \ldots \ldots \ldots$

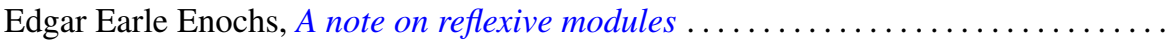

Vladimir Filippenko, On the reflection of harmonic functions and of solutions of the

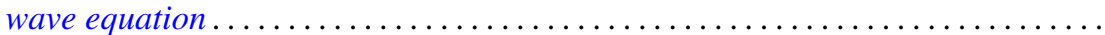

Derek Joseph Haggard Fuller, Mappings of bounded characteristic into arbitrary

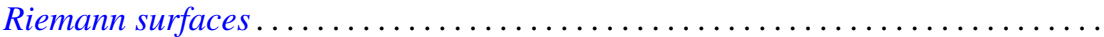
895

Curtis M. Fulton, Clifford vectors . . . . . . . . . . . . . . . . . . . . . . . . . . . . . . 917

Irving Leonard Glicksberg, Maximal algebras and a theorem of Radó . .

919

Kyong Taik Hahn, Minimum problems of Plateau type in the Bergman metric

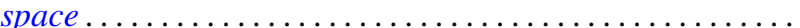

A. Hayes, A representation theory for a class of partially ordered rings...

J. M. C. Joshi, On a generalized Stieltjes trasform

J. M. C. Joshi, Inversion and representation theorems for a generalized Laplace transform ...

Eugene Kay McLachlan, Extremal elements of the convex cone $B_{n}$ of functions ...

Robert Alan Melter, Contributions to Boolean geometry of p-rings ...

James Ronald Retherford, Basic sequences and the Paley-Wiener criterion . . . . . . . 1019

Dallas W. Sasser, Quasi-positive operators. .

Oved Shisha, On the structure of infrapolynomials with prescribed coefficients ..

Oved Shisha and Gerald Thomas Cargo, On comparable means

Maurice Sion, A characterization of weak ${ }^{*}$ convergence ........

Morton Lincoln Slater and Robert James Thompson, A permanent inequality for

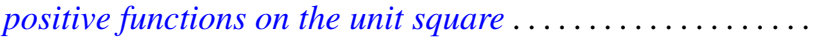

David A. Smith, On fixed points of automorphisms of classical Lie algebras ...

Sherman K. Stein, Homogeneous quasigroups ................

J. L. Walsh and Oved Shisha, On the location of the zeros of some infrapolynomials with prescribed coefficients .

Ronson Joseph Warne, Homomorphisms of $d$-simple inverse semigroups with identity . 